\title{
Developing an Interactive Tabletop Mediated Activity to Induce Collaboration by Implementing Design Considerations Based on Cooperative Learning Principles
}

\author{
Patrick Sunnen $^{1(\bowtie)}$, Béatrice Arend ${ }^{1}$, Svenja Heuser ${ }^{1}$, \\ Hoorieh Afkari ${ }^{2}$, and Valérie Maquil ${ }^{2}$ \\ 1 University of Luxembourg, \\ Porte des Sciences 11, 4366 Esch-sur-Alzette, Luxembourg \\ \{patrick. sunnen, beatrice. arend, svenja.heuser\}@uni.lu \\ ${ }^{2}$ Luxembourg Institute of Science and Technology, \\ Avenue des Hauts-Fourneaux, 4362 Esch-sur-Alzette, Luxembourg \\ \{hoorieh.afkari,valerie.maquil\}@list.lu
}

\begin{abstract}
Constructive collaboration can be a difficult matter. For this reason, we are implementing and studying an interactive-tabletop-mediated activity that aims at inducing collaboration among participants. The resulting activity 'Orbitia' is designed as a serious game. Participants are asked to act as a spacemining crew, which has to collect minerals with a rover and rely on a cameradrone for reconnaissance, while keeping the rover out of harm and managing limited resources. In this paper we provide an account of how we designed Orbitia's pedagogical structuring by relying on the Johnsons' cooperative learning approach whose fundamental concept is "positive interdependence". More particularly, we show how we worked on resource, role and task interdependence to design three collaboration-inducing 'flagship' devices: the roversteering-device (RSD), the item-locating-device (ILD) and the responsibilityactivating-device (RAD).
\end{abstract}

Keywords: Interactive tabletop $\cdot$ Collaboration $\cdot$ Cooperative learning

\section{Introduction}

There is an increasing recognition that societal and intellectual challenges will be (resp. are already) so complicated or complex that they can only be tackled collaboratively. However, constructive collaboration on new challenges is a difficult matter and the mere joining of people's forces does not help unless people know how to collaborate [1]. Hence, learning to learn and to work together must become an important goal in education and professional training. So, the design research project ORBIT (Overcoming Breakdowns in Teams with Interactive Tabletops) aims at implementing and studying a joint problem-solving activity at an interactive tabletop (ITT) [2]. 
In reference to the project name we called this activity 'Orbitia' (see Fig. 1). Orbitia is designed as a serious game where adult participants are asked to act as a spacemining crew. The latter has to collect minerals with a rover and rely on a camera-drone for reconnaissance, while keeping the rover out of harm and managing limited resources. The activity consists of two phases. The first one is designed to induce 'smooth' collaboration among the participants. In the second one, the underlying foundational rules are unexpectedly modified in order to put to the test participants' previously established collaboration procedures. Thus, in order to cope successfully with this advanced dynamic situation, participants will have to re-establish and expand their collaboration procedures.

Stahl [3] rightfully points out that every technology in order to achieve a desirable educational outcome requires "considerable interface design, user evaluation, pedagogical structuring, and collaborative culture" (p. 486). While the implementation of ORBIT's multidisciplinary design-based research process as well as results of the video-taped trials are resp. will be presented elsewhere [4], the purpose of this paper is to provide an account of how a cooperative learning approach $[5,6]$ provided for the pedagogical structuring and informed the design of the now accomplished first phase of Orbitia.

\section{Theoretical Design Considerations}

\subsection{Collaboration}

In everyday life and even in many scientific accounts, the term 'collaboration' is often used very broadly to describe two or more persons working together on the same task. Hence, before we can begin to conceive Orbitia as an interactive tabletop activity inducing collaboration in a face-to-face setting, we have to become more specific about the conduct we seek to solicit here.

In line with a process-oriented meaning-making approach on collaboration [7], we rely on Roschelle and Teasley [8], who define "collaboration" as a "coordinated, synchronous activity that is the result of a continued attempt to construct and maintain a shared conception of a problem" (p. 70). So, the design of Orbitia aims at implementing a co-located activity, that provides a mediational framework supporting participants' mutual engagement, pointing their orientation to a shared goal, facilitating the description of the current problem state, and raising their awareness of problem-solving actions [4].

\subsection{Collaboration and Interactive Tabletops}

Interactive tabletops can integrate physical and digital artefacts, spoken and written texts to provide groups with a focal point and dynamic resource to support collaboration [3]. In other words, they are inherently designed as shareable interfaces (see Fig. 2): their input modalities support more than one participant [9] and their horizontal orientation enables face-to-face communication around a large surface that provides participants with a shared overview and focus point [10]. 


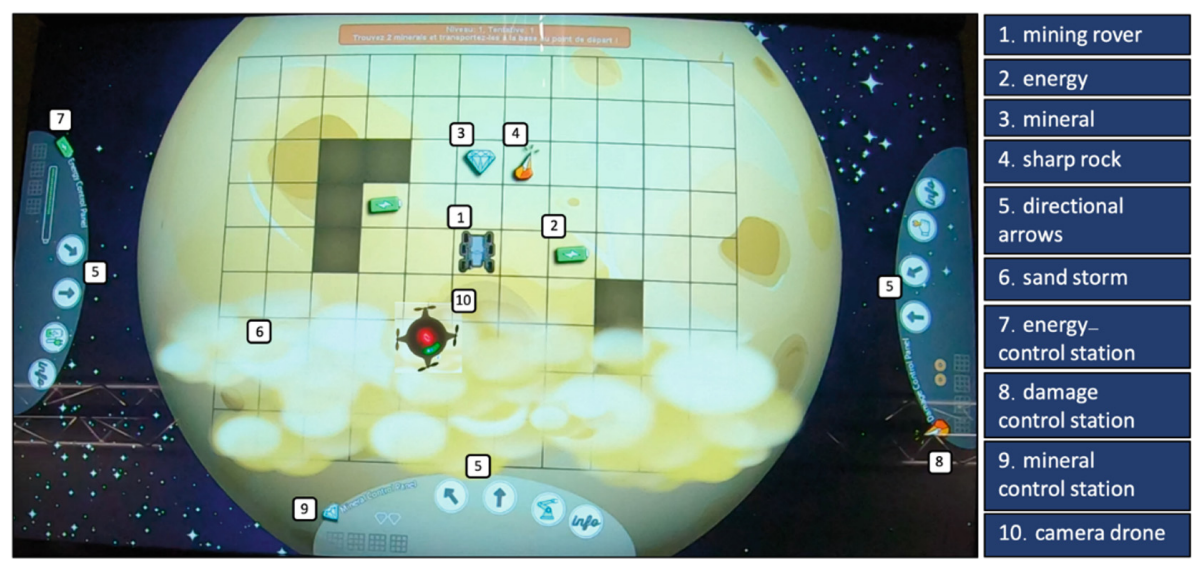

Fig. 1. Annotated screenshot of 'Orbitia'.

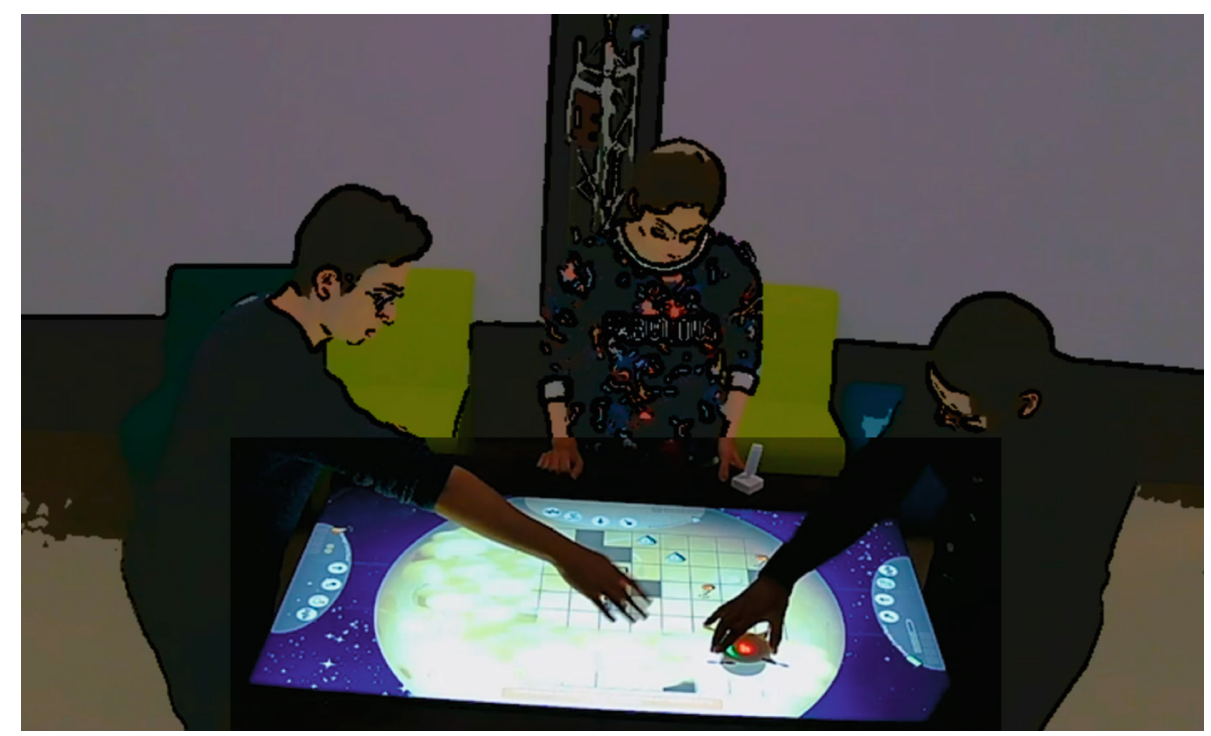

Fig. 2. A group of participants engaging with 'Orbitia'

Pointing to relevant studies Ioannou and Antoniou [11] (p. 165) summarize that tabletops enhance the sense of teamwork, induce interaction and willingness to participate in group tasks, foster equity in physical interaction, promote joint attention on the task, and improve the (learning) experience and engagement with the task. Indeed, when participants' attention is drawn to the tabletop, they can see each other's actions as well as the system's feedback, potentially changing the nature of the collaboration: for example, the explicit awareness of other's hand actions facilitates exploration and increases collaborative forms of construction and meaning making [12] (p. 319). 
However, none of these promising study outcomes can be taken for granted and, as we pointed out above, the pedagogical framework that is designed into the ITTmediated activity is a crucial issue here. For this reason, we shall now outline the essential features of Orbitia's pedagogical structuring and how we implemented them.

\subsection{Cooperative Learning}

In instructional contexts "cooperative learning" and "collaborative learning" are either used interchangeably or presented as having considerable overlap. At times, cooperative learning is considered as the more structured approach where "the structure is imposed by the teacher" and "is designed to achieve a specific goal or end-product" [13] (p. 71). During our literature research we found that a number of researchers [14, 15] take inspiration in Johnson \& Johnson's work on cooperative learning [5, 6] to develop educational collaborative game scenarios. The latter are defined as games where "all the participants work together as a team, sharing the pay-offs and outcomes; if the team wins or loses, everyone wins or loses" [16] (p. 25).

The Johnsons rely on a social interdependence perspective and "cooperation" is described as "positive interdependence", which exists "when students perceive that they are linked with groupmates in a way that makes it impossible for anyone to succeed unless the entire group succeeds (and vice versa) and that they must coordinate their efforts with their groupmates to complete a task" [5] (p. 27). Research indicates that participants engaged in cooperative settings spend considerable time on task, show positive attitudes toward the experience of working on the task, take accurate perspectives, generate new ideas and readily transfer their cooperative efforts to new tasks [17].

Such a defined approach of cooperative learning is suitable for creating a groupworthy task which calls for participants to rely on multiple resources that cannot be mobilised by one person alone, and aims at creating a situation in which participants "work interdependently and reciprocally, and where the exchange of ideas and information" and "the joint construction of understanding" are key to success [18] (p. 115).

In order to construct the pedagogical structuring of 'Orbitia' we relied on the following interdependence principles of "cooperative learning": fantasy (the task is embedded in a compelling narrative, here: space-mining), environmental (group members are bound by the physical environment; here: the tabletop and the graphical representation of a planetary surface), identity (establishment of a group identity, here: participants are positioned as a space mining crew by the narrative), goal (only the group can accomplish the set goal, here: collecting the minerals), task (each participant has to complete a sub-task for the group goal to be achieved, here: steering and retrieving manoeuvres), role (complementary task-relevant responsibilities are distributed among the participants, here: being in charge of mining, repairing or energy matters), resource (every participant holds only a portion of the needed resources, here: for example, individually allocated steering options) and reward (every participant receives the same reward when the group goal is reached, here: moving to the next level and eventually winning the game). To avoid putting pressure on an emerging group, we have not yet implemented an outside enemy interdependence (the group is put in competition with other groups, standards or time). Nevertheless, this feature may 
be applied in the second phase of the activity where the collaboration procedures established by the participants are to be challenged.

\section{Implementing Cooperative Learning Design Considerations}

More particularly, we worked on resource, role and task interdependence to guide the design of three collaboration-inducing 'flagship' devices: the rover-steering-device (RSD), the item-locating-device (ILD) and the responsibility-activating-device (RAD).

(1) The rover-steering-device consists of a virtual manoeuvrable rover with distributed control buttons. Every control station is equipped with two touch-controlled arrows (Fig. 1, no 5; Fig. 3a) enabling each participant to steer the rover into two different directions. This particular distribution of the control options aims at ensuring that every participant has to bring in a specific resource (two unique directions) assigned to him/her in order to move the group's rover to a targeted spot. The steering movements have to be performed in an appropriate order and thus every movement correspond to sub-tasks to be done by the responsible participants. This is reinforced by the fact that two directions (see Fig. 3b) can only be taken through the composition of two other directions, thus requiring the intervention of two participants in close coordination. The distribution of the steering options contributes to the establishment of roles, further strengthened through the organisation of the ILD and the RAD.

(2) The item-locating-device consists of one tangible shared object (drone) with an integrated display, a surrounding $3 \times 3$ digital grid and four mini-maps in each control station (see Fig. 4). The drone can be operated by every participant to disclose the hidden items in the sandstorm area. While being moved over the surface the display shows the number of (hidden) items in the surrounding grid. After pushing a button, the nature of the surrounding items (mineral, energy stack, sharp rock) and their location is briefly revealed in the grid. This information is then distributed to the corresponding mini-maps ${ }^{1}$ in the three control stations. Thus, every participant has a particular complementary resource at his/her disposal, i.e. the information on the location and the amount of the type of items that are associated with his/her control station. This feature aims at supporting the establishment of a specific role for every participant, which is materialised in the RAD.

(3) The responsibility-activating-device offers three different positions materialized as energy, damage, and mineral control stations located in three separate places (Fig. 1, no. 7-9; Fig. 5). Every participant is expected to take the responsibility of one area: energy, mining, or damage management). According to the assumed responsibility, s/he has to monitor the number of attributed items (energy units, number of spare wheels or number of minerals collected) and the corresponding locations of the collectable (energy, minerals) and dangerous items (rocks). The

\footnotetext{
${ }^{1}$ This feature is inspired by the quadrants in the game "Chase the Cheese" [15].
} 


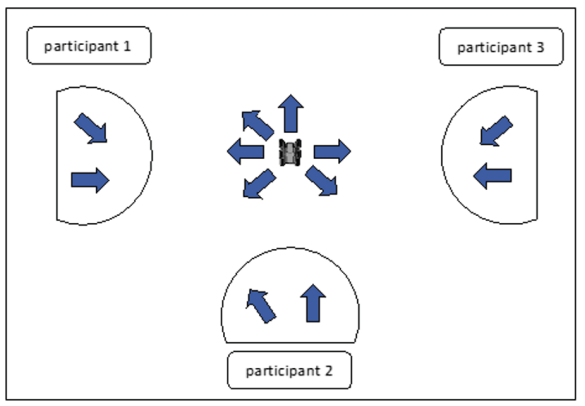

(a)

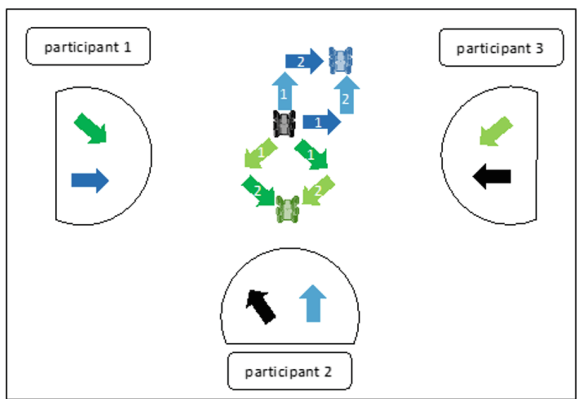

(b)

Fig. 3. (a) Distribution of directions (b) Composing missing directions

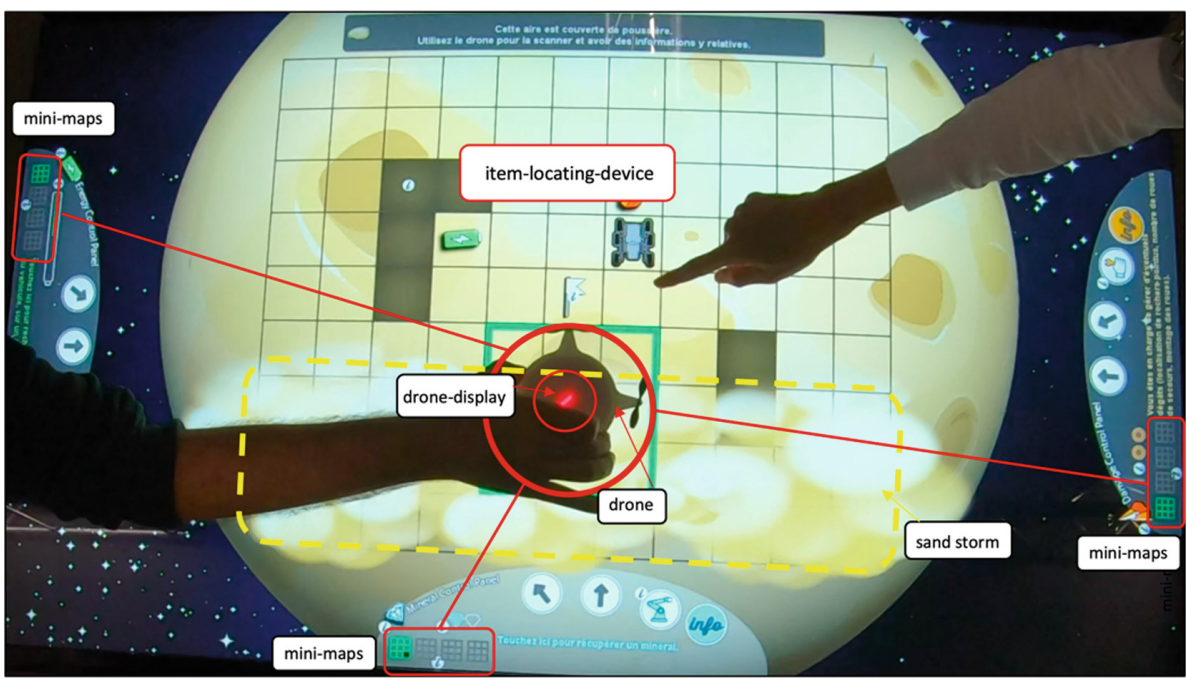

Fig. 4. Participants applying the item-locating-device

different items are marked on the respective mini-maps as dots meaning that the dots appearing in the mini-maps of the mining control station point to the location of the minerals and so on. Thus, according to his/her role, every participant has a particular complementary resource at his/her disposal, which is the information on the location and the amount of the type of items that are associated with his/her control station. This role- or responsibility-related distribution also applies to the information shown in the display area of every control station (Fig. 5, blue rounded rectangle). On pushing the info button first (Fig. 5, red circle) and then the appearing crosses (not visible in the figures), the participant receives responsibility- 


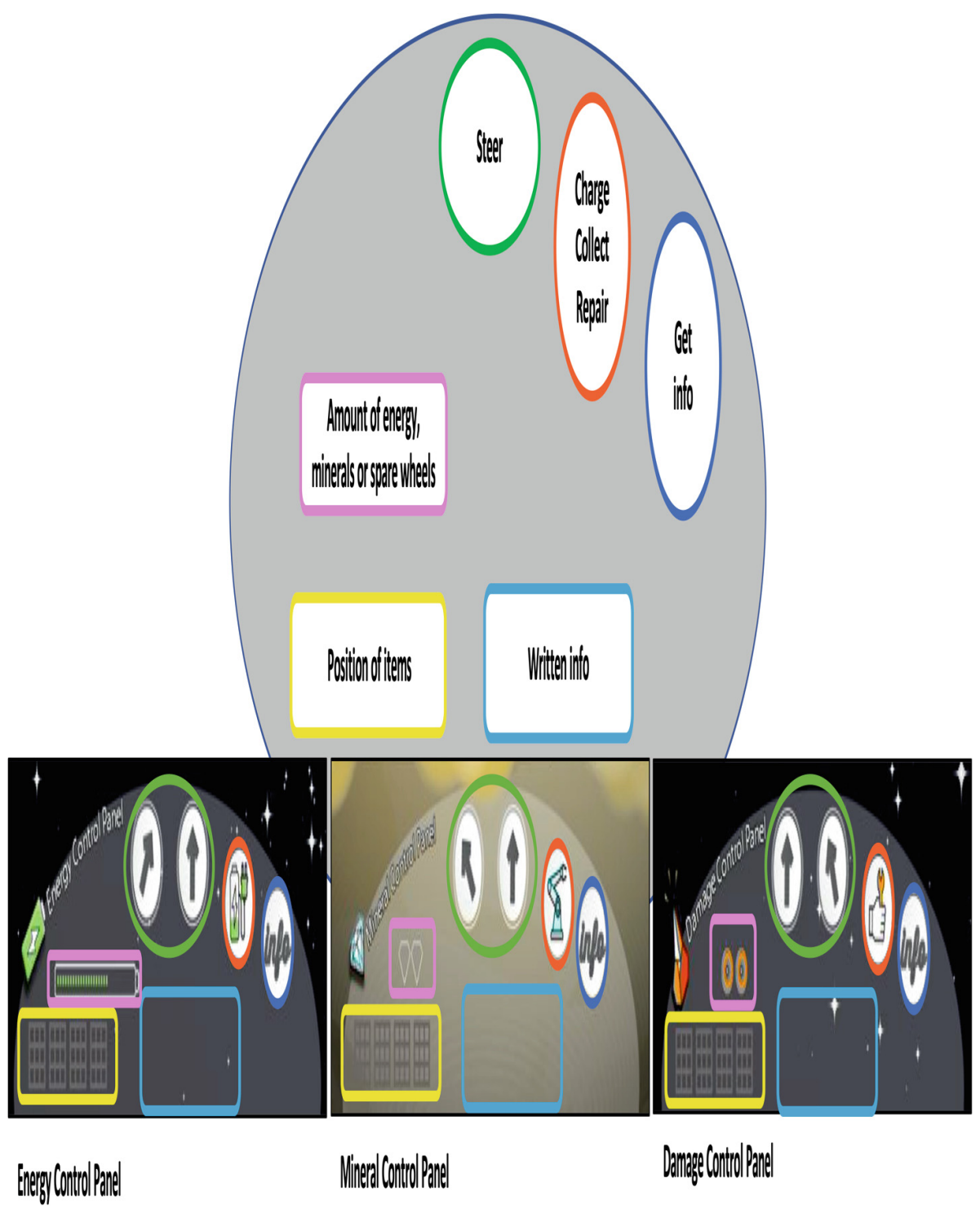

Fig. 5. Responsibility-activating-device (Color figure online)

specific written information ${ }^{2}$ with regard to the features of his/her station. Furthermore, every participant has to perform the related pick-up respectively the

${ }^{2}$ Other less specific information - for example, the mission objective, some features of the map (base, sandstorm, etc.) - is available right away for every participant in a display field above the grid or around the drone. For reasons of space and to ensure better readability, we chose not to elaborate here on how we provided the participants with written operating instructions. 
wheel-changing action by pushing the button on his/her control station provided for this purpose (task interdependence).

\section{Conclusion}

Taking Stahl's [3] caution seriously, we conceptualised the pedagogical design of our collaboration-inducing ITT-mediated activity Orbitia by relying on a sound and validated cooperative learning approach developed by the Johnsons [5, 6]. We are aware that all the other considerations listed by Stahl [3] - interface design, user evaluation and collaboration culture - are equally important and closely intertwined. That's why we plan to address these issues in subsequent publications. In this paper, we elaborated on how we implemented the concept of "positive interdependence" [5,6] in the design of Orbitia. So far, our ongoing user evaluation and video analyses indicate that participants experience the engagement with Orbitia as collaborative, display collaborative conduct in the sense of [8], and actively engage with and appropriate the three developed flagship devices the rover-steering-device, the item-locating-device and the responsibility-activating-device.

Acknowledgements. We thank the Luxembourg National Research Fund (FNR) for funding this research under the CORE scheme.

\section{References}

1. Schwarz, B.B., de Grootet, R., Mavrikis, M., Dragon, T.: Learning to learn together with CSCL tools. Int. J. Comput.-Supported Collab. Learn. 10(3), 239-271 (2015)

2. Sunnen, P., Arend, B., Maquil, V.: ORBIT - overcoming breakdowns in teams with interactive tabletops. In: Rethinking Learning in the Digital Age: Making the Learning Sciences Count, 13th International Conference of the Learning Sciences (ICLS) 2018, London (2018)

3. Stahl, G.: Collaborating around the tabletop. Int. J. Comput. Support. Collab. Learn. 6(4), 485-490 (2011)

4. Sunnen, P., Arend, B., Heuser, S., Afkari, H., Maquil, V.: Designing collaborative scenarios on tangible tabletop interfaces - insights from the implementation of paper prototypes in the context of a multidisciplinary design workshop. In: 17th European Conference on ComputerSupported Cooperative Work, Salzburg (2019)

5. Johnson, D.W., Johnson, R.T., Johnson Holubec, E.: The New Circles of Learning. Cooperation in the Classroom. ASCD, Alexandria (1994)

6. Johnson, D., Johnson, R.: Positive Interdependence. The Heart of Cooperative Learning. Interaction Book Company, Edina (1992)

7. Stahl, G., Koschmann, T., Suthers, D.: Computer-supported collaborative learning. In: Sawyer, R.K. (ed.) The Cambridge Handbook of the Learning Sciences, 2nd edn, pp. 479499. Cambridge University Press, New York (2014)

8. Roschelle, J., Teasley, S.: The construction of shared knowledge in collaborative problem solving. In: O’Malley, C. (ed.) Computer Supported Collaborative Learning, pp. 69-97. Springer, Berlin (1995). https://doi.org/10.1007/978-3-642-85098-1_5 
9. Evans, M.A., Rick, J.: Supporting learning with interactive surfaces and spaces. In: Spector, J.M., Merill, M.D., Elen, J., Bishop, M. (eds.) Handbook of Research on Educational Communications and Technology, pp. 689-701. Springer, New York (2014). https://doi.org/ 10.1007/978-1-4614-3185-5_55

10. Brudy, F., Marquard, N.: The tabletop is dead? - long live the table's top! submitted to the workshop: "the disappearing tabletop - social and technical challenges for cross-surface collaboration". In: conjunction with the ACM Conference on Interactive Surfaces and Spaces 2017. ISS, Brighton (2017)

11. Ioannou, A., Antoniou, C.: Tabletops for peace: technology enhanced peacemaking in school contexts. Educ. Technol. Soc. 19(2), 164-176 (2016)

12. Price, S.: Tangibles: technologies and interaction for learning. In: Price, S., Jewitt, C., Brown, B. (eds.) The Sage Handbook of Digital Technology Research, pp. 307-325. Sage, London (2013)

13. McWhaw, K., Schnackenberg, H., Sclater, J., Arambi, P.-C.: From co-operation to collaboration. In: Gillies, R., Brown, B. (eds.) Co-operative Learning, pp. 307-325. Sage, London, New York (2003)

14. Oskanen, K., Hämäläinen, R.: Game mechanics in the design of a collaborative 3D serious game. Simul. Gaming 45(2), 255-278 (2014)

15. Collazos, C.A., Guerrero, L.A., Pino, J.A., Ochoa, S.F.: Collaborative scenarios to promote positive interdependence among group members. In: Favela, J., Decouchant, D. (eds.) CRIWG 2003. LNCS, vol. 2806, pp. 356-370. Springer, Heidelberg (2003). https://doi.org/ 10.1007/978-3-540-39850-9_30

16. Zagal, J.P., Rick, J., Hsi, I.: Collaborative games: lessons learned from board games. Simul. Gaming 37(1), 24-40 (2006)

17. Johnson, D.W., Johnson, R.T.: Cooperative, competitive, and individualistic learning environments. In: Hattie, J., Anderman, E.M. (eds.) International Guide to Student Achievement, pp. 372-374. Routledge, New York (2013)

18. Vass, E., Littleton, K.: Peer collaboration and learning in the classroom. In: Littleton, K., Wood, C., Staarman, K.J. (eds.) International Handbook of Psychology in Education, pp. 105-135. Emerald Group Publishing Limited, Bingley (2010)

Open Access This chapter is licensed under the terms of the Creative Commons Attribution 4.0 International License (http://creativecommons.org/licenses/by/4.0/), which permits use, sharing, adaptation, distribution and reproduction in any medium or format, as long as you give appropriate credit to the original author(s) and the source, provide a link to the Creative Commons license and indicate if changes were made.

The images or other third party material in this chapter are included in the chapter's Creative Commons license, unless indicated otherwise in a credit line to the material. If material is not included in the chapter's Creative Commons license and your intended use is not permitted by statutory regulation or exceeds the permitted use, you will need to obtain permission directly from the copyright holder.

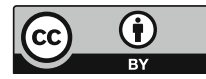

